\title{
Tropospheric delay parameters from numerical weather models for multi-GNSS precise positioning
}

\author{
Cuixian Lu ${ }^{1}$, Florian Zus ${ }^{1}$, Maorong Ge ${ }^{1}$, Robert Heinkelmann ${ }^{1}$, Galina Dick ${ }^{1}$, Jens Wickert ${ }^{1,2}$, and Harald Schuh ${ }^{1,2}$ \\ ${ }^{1}$ German Research Centre for Geosciences GFZ, Telegrafenberg, 14473 Potsdam, Germany \\ ${ }^{2}$ Technische Universität Berlin, Institute of Geodesy and Geoinformation Science, 10623 Berlin, Germany
}

Correspondence to: Cuixian Lu (cuixian@gfz-potsdam.de)

Received: 23 May 2016 - Published in Atmos. Meas. Tech. Discuss.: 16 June 2016

Revised: 17 November 2016 - Accepted: 24 November 2016 - Published: 13 December 2016

\begin{abstract}
The recent dramatic development of multi-GNSS (Global Navigation Satellite System) constellations brings great opportunities and potential for more enhanced precise positioning, navigation, timing, and other applications. Significant improvement on positioning accuracy, reliability, as well as convergence time with the multi-GNSS fusion can be observed in comparison with the single-system processing like GPS (Global Positioning System). In this study, we develop a numerical weather model (NWM)-constrained precise point positioning (PPP) processing system to improve the multi-GNSS precise positioning. Tropospheric delay parameters which are derived from the European Centre for Medium-Range Weather Forecasts (ECMWF) analysis are applied to the multi-GNSS PPP, a combination of four systems: GPS, GLONASS, Galileo, and BeiDou. Observations from stations of the IGS (International GNSS Service) Multi-GNSS Experiments (MGEX) network are processed, with both the standard multi-GNSS PPP and the developed NWM-constrained multi-GNSS PPP processing. The high quality and accuracy of the tropospheric delay parameters derived from ECMWF are demonstrated through comparison and validation with the IGS final tropospheric delay products. Compared to the standard PPP solution, the convergence time is shortened by $20.0,32.0$, and $25.0 \%$ for the north, east, and vertical components, respectively, with the NWM-constrained PPP solution. The positioning accuracy also benefits from the NWM-constrained PPP solution, which was improved by $2.5,12.1$, and $18.7 \%$ for the north, east, and vertical components, respectively.
\end{abstract}

\section{Introduction}

As the first space-based satellite navigation system, Global Positioning System (GPS) consisting of a dedicated satellite constellation has been extensively applied for many geodetic applications in the last decades. In particular, the GPS precise point positioning (PPP, Zumberge et al., 1997) method draws special interest as it enables accurate positioning of millimeter to centimeter accuracy with a single receiver (Blewitt et al., 2006). Due to its significant advantages in terms of operational flexibility, global coverage, cost efficiency, and high accuracy, the PPP approach has been demonstrated to be a powerful tool and it is widely used in various fields such as precise orbit determination (POD) of low-Earth orbiter (LEO), crustal deformation monitoring, precise timing, GPS meteorology, and kinematic positioning of mobile platforms (Zumberge et al., 1997; Kouba and Héroux, 2001; Gao and Shen, 2001; Zhang and Andersen, 2006; Ge et al., 2008). With the continuously improved density of the tracking network infrastructure as well as the enhanced precise satellite orbit and clock correction products with shortlatency (e.g., real-time) availability, many innovative applications like geo-hazard monitoring, seismology, nowcasting of severe weather events or regional short-term forecasting based on the PPP technique have also been emerging and undergoing great developments (Larson et al., 2003; Li et al., 2013; Lu et al., 2015). However, the GPS-only PPP shows limitations concerning the convergence time, positioning accuracy, and long re-initialization period due to insufficient satellite visibility and limited spatial geometry, especially under constrained environmental conditions where the signals are blocked or interrupted. 
The world of satellite navigation is going through dramatic changes and is stepping onto a stage of multi-constellation GNSS (Global Navigation Satellite System) (Montenbruck et al., 2014). Not only is GPS of full capability and under continuous modernization, but also GLONASS has finished the revitalization and is now fully operational. Besides, two new constellations, Galileo and BeiDou, have recently emerged. The European Galileo currently comprises 12 satellites deployed in orbit and it is working towards a fully operational stage. The Chinese BeiDou officially launched a continuous positioning, navigation, and timing (PNT) service covering the whole Asia Pacific region at the end of 2012. It is continuously developing to a global system in the near future. In addition, the Japanese Quasi-Zenith Satellite System (QZSS) and the Indian Regional Navigation Satellite System (IRNSS) are also growing, with one and five satellites currently (as of 2016) operating in orbit, respectively. So far, more than 80 navigation satellites can be in view and transmit data benefitting from the multi-constellation GNSS, which brings great opportunities for more precise positioning, navigation, timing, remote sensing, and other applications (Ge et al., 2012).

Undoubtedly, the integration of all existing navigation satellite systems could provide more observations and could thus enable definite improvements on reliability, positioning accuracy, and convergence time of PPP in comparison with the stand-alone GPS PPP. Li et al. (2015a) developed a foursystem (GPS + GLONASS + Galileo + BeiDou) positioning model to fully exploit all available observables from different GNSS. They demonstrated that the fusion of multiple GNSS showed a significant effect on shortening the convergence time and improving the positioning accuracy when compared to single-system PPP solutions. The benefits of the four-system model were also found when applied for realtime precise positioning ( $\mathrm{Li}$ et al., 2015b), where a reduction of the convergence time by about $70 \%$ and an improvement of the positioning accuracy by about $25 \%$ with respect to the GPS-only processing were illustrated. The fusion of multi-GNSS constellations has developed to be one of the hot topics within the GNSS community, not only limited to precise positioning but also for related applications. For example, the multi-GNSS PPP exhibits significant advantages for GNSS meteorology applications, such as the real-time retrieval of atmospheric parameters including integrated water vapor, tropospheric delays, and horizontal gradients, in particular for the high-temporal resolution tropospheric gradients (Li et al., 2015c; Lu et al., 2016). Therefore, improving the performance of multi-GNSS precise positioning concerning both positioning accuracy and solution convergence is the main focus of our study.

Numerical weather models (NWMs) are able to provide the required information for describing the neutral atmosphere, from which the meteorological parameters can be derived at any location and at any time by applying interpolation, within the area and time window considered by the model (Pany et al., 2001). In the past, the application of NWM in space geodetic analysis mainly focused on the determination of mapping functions (Niell, 1996; Boehm et al., 2006). With respect to the improvements in spatiotemporal resolutions as well as in precision and accuracy of the NWM during recent years, tropospheric delay parameters, such as zenith total delay (ZTD), slant total delays, and tropospheric gradients, derived from the NWM could satisfy the accuracy requirements for most GNSS applications (Andrei and Chen, 2008). Data from the NWM have been used to perform tropospheric delay modeling or correct for the neutral atmospheric effects in GNSS data processing. Hobiger et al. (2008a) made use of ray-traced slant total delays derived from a regional NWM for GPS PPP within the area of eastern Asia. They demonstrated an improvement of station coordinate repeatability by using this strategy in comparison to the standard PPP approach where the tropospheric delays were estimated as unknown parameters. Furthermore, an enhanced algorithm for extracting the ray-traced tropospheric delays of higher accuracy from the NWM in real-time mode was proposed by Hobiger et al. (2008b). The authors presented the potential and the feasibility of applying the NWM-derived tropospheric delay corrections into real-time PPP processing. Besides, Ibrahim and El-Rabbany (2011) evaluated the performance of implementing tropospheric corrections from the NOAA (National Oceanic and Atmospheric Administration) Tropospheric Signal Delay Model (NOAATrop) into GPS PPP. They pointed out an improvement of convergence time by about 1,10 , and $15 \%$ for the latitude, longitude, and height components, respectively, by using the NOAA troposphere model when compared to the results achieved with the previously used Hopfield model.

In this study, we develop a NWM-constrained PPP processing method to improve the multi-GNSS (a combination of four systems: GPS, GLONASS, Galileo, and BeiDou) precise positioning. Tropospheric delay parameters, which are derived from the European Centre for MediumRange Weather Forecasts (ECMWF, http://www.ecmwf.int/) analysis, are applied to multi-GNSS PPP. Observations from the IGS (International GNSS Service) Multi-GNSS Experiments (MGEX) network are processed. The quality of tropospheric delay parameters retrieved from the ECMWF analysis is assessed by comparison with the IGS final tropospheric delay products (ftp://cddis.gsfc.nasa.gov/ gnss/products/troposphere/zpd/). The performance of multiGNSS PPP making use of the NWM-derived tropospheric delay parameters is evaluated in terms of both convergence time and positioning accuracy.

This article is organized as follows: Sect. 2 illustrates the IGS tracking network for MGEX, the multi-GNSS data collection, and the tropospheric delay parameters retrieved from ECMWF. Two multi-GNSS PPP processing scenarios - the standard and the NWM-constrained PPP - are presented in detail focusing on the modeling of the tropospheric delays. Thereafter, Sect. 3 describes the comparison of tropospheric 
delay parameters from ECMWF with respect to the IGS final tropospheric delay products. In Sect. 3, the positioning results, in terms of the convergence time and the positioning accuracy, achieved with the NWM-constrained multi-GNSS PPP solution are illustrated in comparison to the ones with the standard PPP solution. The conclusions and discussions are presented in Sect. 4.

\section{Data collection and processing}

\subsection{Multi-GNSS data collection}

In response to the dramatic development of the global satellite navigation world along with the upcoming systems and signals, the IGS initialized the MGEX campaign to enable a multi-GNSS service of tracking, collecting, and analyzing data of all available signals from GPS, GLONASS, BeiDou, Galileo, QZSS, and any other space-based augmentation system (SBAS) of interest (Montenbruck et al., 2014). Accordingly, a new worldwide network of multi-GNSS monitoring stations under the framework of the MGEX project has been deployed in the past 2 years in parallel with the IGS network, which only serves for GPS and GLONASS. Currently, the MGEX network consists of more than 120 stations, which are globally distributed and provide excellent capability of multi-GNSS constellation tracking and data delivering owing to the contributions from about 27 agencies, universities, and other institutions of 16 countries (http://igs.org/mgex). Besides the tracking of the GPS constellation, the majority of the MGEX stations offer the GLONASS data. At least one of the new BeiDou, Galileo, or QZSS constellations can be tracked for each MGEX station. Today, about 75 stations are capable of tracking the Galileo satellites, 80 stations are tracking the GLONASS satellites, and the BeiDou constellation is supported by more than 30 receivers. Figure 1 shows the geographical distribution of the MGEX stations and their supported constellations, except GPS, which can be tracked by each station.

\subsection{NWM data collection}

The pressure, temperature, and specific humidity fields of the ECMWF operational analysis are utilized to retrieve the tropospheric delay parameters. The ECMWF data are available at the German Research Centre for Geosciences (GFZ) with a horizontal resolution of $1^{\circ} \times 1^{\circ}$ on 137 vertical model levels extending from the Earth's surface to about $80 \mathrm{~km}$. We use the ray-trace algorithm proposed by Zus et al. (2014) and compute station-specific zenith hydrostatic (non-hydrostatic) delays, derive all three hydrostatic (non-hydrostatic) mapping function coefficients (Zus et al., 2015a) and the horizontal delay gradient components (Zus et al., 2015b). The calculated station-specific tropospheric delay parameters are available every $6 \mathrm{~h}$ per day and are valid at 00:00, 06:00, 12:00, and 18:00 UTC. These ECMWF-derived tropospheric

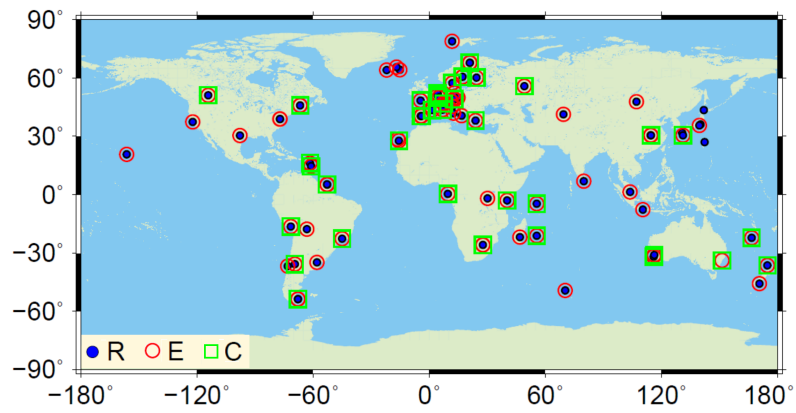

Figure 1. The geographical distribution of the MGEX stations and their supported navigation satellite constellations. R, E, and C refer to GLONASS, Galileo, and BeiDou, respectively, while GPS can be tracked by each station.

delay parameters are linearly interpolated to be applied in the GNSS processing.

\subsection{Multi-GNSS PPP processing}

In the PPP processing, precise satellite orbits and clocks are fixed to previously determined values. The multi-GNSS (here GPS, GLONASS, Galileo, and BeiDou) PPP processing model can be expressed as follows:

$$
\begin{aligned}
& l_{\mathrm{r}_{\mathrm{r}, j}}^{G}=-\boldsymbol{u}_{\mathrm{r}}^{G} \cdot \boldsymbol{r}+t_{\mathrm{r}}+\lambda_{j G}\left(b_{\mathrm{r} G, j}-b_{j}^{G}\right)+\lambda_{j G} N_{\mathrm{r}, j}^{G}-\kappa_{j G} \cdot I_{\mathrm{r}, 1}^{G}+T+\varepsilon_{\mathrm{r}, j}^{G} \\
& l_{\mathrm{r}, j}^{R_{k}}=-\boldsymbol{u}_{\mathrm{r}}^{R} \cdot \boldsymbol{r}+t_{\mathrm{r}}+\lambda_{j R_{k}}\left(b_{\mathrm{r} R_{k}, j}-b_{j}^{R}\right)+\lambda_{j R_{k}} N_{\mathrm{r}, j}^{R}-\kappa_{j R_{k}} \cdot I_{\mathrm{r}, 1}^{R}+T+\varepsilon_{\mathrm{r}, j}^{R} \\
& l_{\mathrm{r}, j}^{E}=-\boldsymbol{u}_{\mathrm{r}}^{E} \cdot \boldsymbol{r}+t_{\mathrm{r}}+\lambda_{j E}\left(b_{\mathrm{r} E, j}-b_{j}^{E}\right)+\lambda_{j E} N_{\mathrm{r}, j}^{E}-\kappa_{j E} \cdot I_{\mathrm{r}, 1}^{E}+T+\varepsilon_{\mathrm{r}, j}^{E} \\
& l_{\mathrm{r}, j}^{C}=-\boldsymbol{u}_{\mathrm{r}}^{C} \cdot \boldsymbol{r}+t_{\mathrm{r}}+\lambda_{j C}\left(b_{\mathrm{r} C, j}-b_{j}^{C}\right)+\lambda_{j C} N_{\mathrm{r}, j}^{C}-\kappa_{j C} \cdot I_{\mathrm{r}, 1}^{C}+T+\varepsilon_{\mathrm{r}, j}^{C} \\
& \left\{\begin{array}{l}
p_{\mathrm{r}, j}^{G}=-\boldsymbol{u}_{\mathrm{r}}^{G} \cdot \boldsymbol{r}+t_{\mathrm{r}}+c \cdot d_{\mathrm{r} G}+\kappa_{j G} \cdot I_{\mathrm{r}, 1}^{G}+T+e_{\mathrm{r}, j}^{G} \\
p_{\mathrm{r}_{,}, j}^{R}=-\boldsymbol{u}_{\mathrm{r}}^{R} \cdot \boldsymbol{r}+t_{\mathrm{r}}+c \cdot d_{\mathrm{r} R_{k}}+\kappa_{j} R_{k} \cdot I_{\mathrm{r}, 1}^{R}+T+e_{\mathrm{r}, j}^{R} \\
p_{\mathrm{r}, j}^{E}=-\boldsymbol{u}_{\mathrm{r}}^{E} \cdot \boldsymbol{r}+t_{\mathrm{r}}+c \cdot d_{\mathrm{r} E}+\kappa_{j E} \cdot I_{\mathrm{r}, 1}^{E}+T+e_{\mathrm{r}, j}^{E} \\
p_{\mathrm{r}, j}^{C}=-\boldsymbol{u}_{\mathrm{r}}^{C} \cdot \boldsymbol{r}+t_{\mathrm{r}}+c \cdot d_{\mathrm{r} C}+\kappa_{j C} \cdot I_{\mathrm{r}, 1}^{C}+T+e_{\mathrm{r}, j}^{C}
\end{array}\right.
\end{aligned}
$$

where $\mathrm{r}$ and $j$ refer to receiver and frequency, respectively; the capital indices $G, R, E$, and $C$ represent the satellites of GPS, GLONASS, Galileo, and BeiDou, respectively; $R_{k}$ denotes the GLONASS satellite with frequency factor $k ; l_{\mathrm{r}, j}$ and $p_{\mathrm{r}, j}$ denote the "observed minus computed" phase and pseudo-range observables; $\boldsymbol{u}_{\mathrm{r}}^{\mathrm{s}}$ is the unit vector in the receiver to satellite direction; $\boldsymbol{r}$ denotes the vector of the receiver position increments relative to the a priori position, which is used for linearization; $t_{\mathrm{r}}$ is the receiver clock bias; $N_{\mathrm{r}, j}$ is the integer ambiguity; $b_{\mathrm{r}, j}$ and $b_{j}^{\mathrm{s}}$ are the uncalibrated phase delays for receivers and satellites, respectively; $\lambda_{j}$ is the wavelength; the ionospheric delays $I_{\mathrm{r}, j}$ at different frequencies can be expressed as $I_{\mathrm{r}, j}=\kappa_{j} \cdot I_{1}, \kappa_{j}=\lambda_{j}^{2} / \lambda_{1}^{2}$; and $T$ is the slant tropospheric delay. Due to the different frequencies and signal structures of each individual GNSS, the code biases $d_{\mathrm{r} G}, d_{\mathrm{r} R_{k}}, d_{\mathrm{r} E}$, and $d_{\mathrm{r} C}$ are different for each multiGNSS receiver. These inter-system biases (ISBs) and interfrequency biases (IFBs) of the GLONASS satellites with different frequency factors have to be estimated or corrected for a combined processing of multi-GNSS observations. $e_{\mathrm{r}, j}$ 
and $\varepsilon_{\mathrm{r}, j}$ denote the sum of measurement noise and multipath effects of pseudo-range and phase observations, respectively. The phase center offsets and variations, the tidal loading, and the phase wind-up are corrected with the models according to Kouba (2009).

The slant total delay $T$ can be described as the sum of the hydrostatic and non-hydrostatic/wet components, and the horizontal gradient components (Chen and Herring, 1997):

$$
\begin{aligned}
T & =\mathrm{mf}_{\mathrm{h}} \cdot \mathrm{ZHD}+\mathrm{mf}_{\mathrm{nh}} \cdot \mathrm{ZWD}+\mathrm{mf}_{\mathrm{G}} \cdot\left(G_{\mathrm{ns}} \cdot \cos (a)\right. \\
& \left.+G_{\mathrm{ew}} \cdot \sin (a)\right)
\end{aligned}
$$

where ZHD and ZWD denote the zenith hydrostatic and nonhydrostatic/wet delays, respectively, $\mathrm{mf}_{\mathrm{h}}$ and $\mathrm{mf}_{\mathrm{nh}}$ are the hydrostatic and non-hydrostatic mapping functions (here global mapping functions, GMFs; Boehm et al., 2006), $\mathrm{mf}_{\mathrm{G}}$ represents the gradient mapping function, $G_{\mathrm{ns}}$ and $G_{\mathrm{ew}}$ are the north-south and east-west delay gradients, respectively, and $a$ is the azimuth of the line of sight of the individual observation.

Concerning the approach for tropospheric delay modeling, two PPP scenarios are applied in this study: one is the standard PPP processing with tropospheric delays estimated as unknown parameters, and the other is the developed NWMconstrained PPP algorithm which utilizes tropospheric delay parameters derived from ECMWF. For the standard PPP processing, a priori ZHD is calculated by use of the empirical models (Saastamoinen, 1973) based on the provided meteorological information (here Global Pressure and Temperature 2 model, GPT2; Lagler et al., 2013) at a given location. Owing to the high variability of the water vapor distribution, the ZWD is estimated as an unknown parameter in the adjustment together with the other parameters, such as the station coordinates. The horizontal tropospheric gradients, $G_{\mathrm{ns}}$ and $G_{\text {ew }}$, are also estimated, both with a temporal resolution of $24 \mathrm{~h}$. The parameters estimated in the standard PPP processing include station coordinates, ambiguity parameters, receiver clock corrections, ZWD, and gradient components, all of which are adjusted in a sequential least squares filter. For the standard multi-GNSS PPP processing, the parameter vector $\boldsymbol{X}$ can be described as

$\boldsymbol{X}=\left(\boldsymbol{r} t_{\mathrm{r}} \mathrm{ZWD} G_{\mathrm{ns}} G_{\mathrm{ew}} d_{\mathrm{r} E} d_{\mathrm{r} C} d_{\mathrm{r} R_{k}} \boldsymbol{I}_{\mathrm{r}, 1}^{\mathrm{s}} \boldsymbol{N}_{\mathrm{r}, j}^{\mathrm{s}}\right)^{T}$.

For the NWM-constrained PPP approach, ZHD, hydrostatic and non-hydrostatic mapping functions are derived from the ECMWF analysis. The ZWD from ECMWF is considered as the a priori value for the wet delays, while a residual wet delay is estimated during the parameter estimation process in order to account for possible imperfections inherent in the NWM. The horizontal gradients are also derived from the ECMWF analysis and are fixed during the processing. In this approach, the unknown parameters are station coordinates, ambiguity parameters, receiver clock corrections, and the residual ZWD. The latter is modeled as a random-walk process with a noise intensity of $5 \mathrm{~mm} \sqrt{ } \mathrm{h}^{-1}$ and a priori constraints. The constraints of the residual ZWD is referred to the accuracy of ECMWF-derived parameters with respect to the IGS tropospheric products, which is a function of station latitudes as illustrated in Fig. 6. Accordingly, the parameter vector $\boldsymbol{X}$ in the NWM-constrained multi-GNSS PPP can be expressed as

$\boldsymbol{X}=\left(\boldsymbol{r} t_{\mathrm{r}} \operatorname{ResizWD}_{\mathrm{r} E} d_{\mathrm{r} C} d_{\mathrm{r} R_{k}} \boldsymbol{I}_{\mathrm{r}, 1}^{\mathrm{s}} \boldsymbol{N}_{\mathrm{r}, j}^{\mathrm{s}}\right)^{T}$,

where ResizwD denotes the residual ZWD.

In order to carry out a rigorous multi-GNSS analysis including the estimation of the inter-system and interfrequency biases, the observables from the four individual GNSS are processed together in a single weighted least squares estimator. The EPOS-RT software (Ge et al., 2012; Li et al., 2013) is utilized for the GNSS data processing in this study, and the GFZ precise products are used.

For the two multi-GNSS PPP scenarios, the receiver position increment $\boldsymbol{r}$ is estimated as static parameter on a daily basis. The receiver clock bias $t_{\mathrm{r}}$ is estimated as white noise, and the inter-system and inter-frequency code biases are estimated as parameters on a daily basis. The ZWD or the residual wet delay ResizwD is modeled as a random-walk process. The code biases for GPS satellites are set to zero to eliminate the singularity between receiver clock and code bias parameters. All the estimated biases of the other systems are relative to those of the GPS satellites. The a priori noise value of $2 \mathrm{~mm}$ for the phase raw observables and $0.6 \mathrm{~m}$ for the code raw observables are applied for each system. The phase ambiguity parameters $N_{\mathrm{r}, j}^{\mathrm{s}}$, which absorb the phase delays $b_{j}^{\mathrm{s}}$, are estimated as constants for each continuous arc. With the combination of the dual-frequency raw phase and pseudo-range observations, the ionospheric delays $\boldsymbol{I}_{\mathrm{r}, 1}^{\mathrm{s}}$ are considered as estimated parameters for each satellitesite pair and each epoch. Besides, an elevation-dependent weighting $\left(e<30^{\circ}, 2 \times \sin (e)\right.$; otherwise, 1$)$ and a cut-off elevation angle of $5^{\circ}$ are applied.

\section{Results and analysis}

\subsection{Comparison between ECMWF and IGS ZTD}

In this section, the quality of tropospheric zenith delay parameters derived from ECMWF analysis is evaluated by comparing with the zenith path delay products offered by IGS (Byram et al., 2011). Specifically, the ECMWF ZTDs for 34 globally distributed stations from the IGS MGEX network during September 2015 are validated by the official IGS ZTD products which are provided with a temporal resolution of $5 \mathrm{~min}$. As the ECMWF ZTDs are sampled every $6 \mathrm{~h}$, we do not interpolate in time but restrict the comparison to the ECMWF data epochs. 

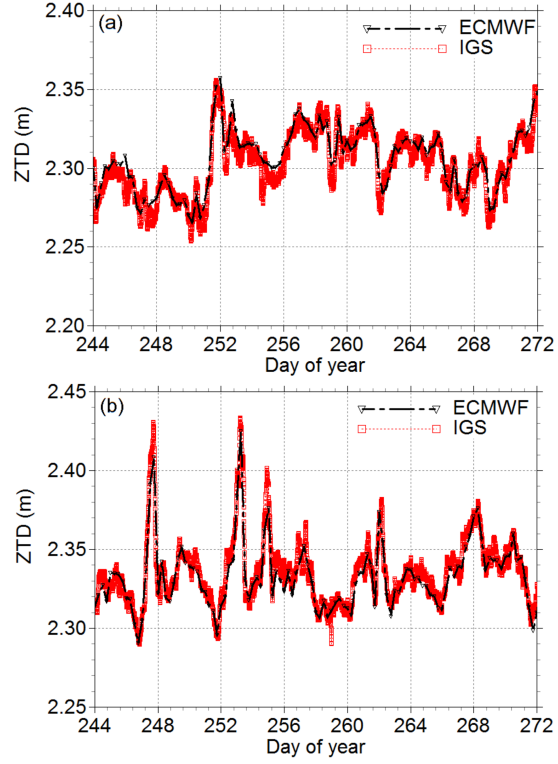

Figure 2. The time series of ECMWF and IGS ZTDs at stations KIRU (a) and NNOR (b) for September (day of year, DOY, 244272) 2015. The ECMWF ZTDs are shown by black triangles, while the IGS ZTDs are displayed by red squares.

As typical examples, the ZTD series derived from ECMWF and IGS at stations KIRU (Kiruna, Sweden) and NNOR (New Norcia, Australia) are shown in Fig. 2. The ECMWF ZTDs are represented by black triangles, while the IGS ZTDs are displayed by red squares. One can notice that the ECMWF ZTDs show good agreement with the IGS ZTDs in general. Most of the peaks in the ZTD series, which are mainly caused by rapid changes of the water vapor content above a station, are captured by ECMWF and IGS solutions.

The corresponding linear correlations between the ECMWF and the IGS ZTDs at stations KIRU and NNOR are illustrated in Fig. 3. It can be seen that ZTDs from the two solutions are highly correlated, with the correlation coefficients being about 0.93 and 0.97 , respectively. Figure 4 presents the distribution of ZTD differences between ECMWF and IGS for the two stations during the same period. One can notice that the ZTD differences mainly range from -15 to $15 \mathrm{~mm}$ for station KIRU, and vary between -10 and $10 \mathrm{~mm}$ for station NNOR. The mean biases of the ZTD differences between the two solutions are -3.52 and $3.31 \mathrm{~mm}$ for the two stations and the root-mean-square (rms) values of the ZTD differences are 8.68 and $6.39 \mathrm{~mm}$, respectively, showing an agreement at the millimeter level.

Figure 5 illustrates the map of station-specific mean biases and rms values of ZTD differences between ECMWF and IGS for all stations. One can notice that the mean biases are within $\pm 15 \mathrm{~mm}$, and that a better agreement between the ECMWF and IGS ZTDs for the high-latitude stations than for the low-latitude stations can be observed. The rms values of the ZTD differences are less than $22 \mathrm{~mm}$, indicating
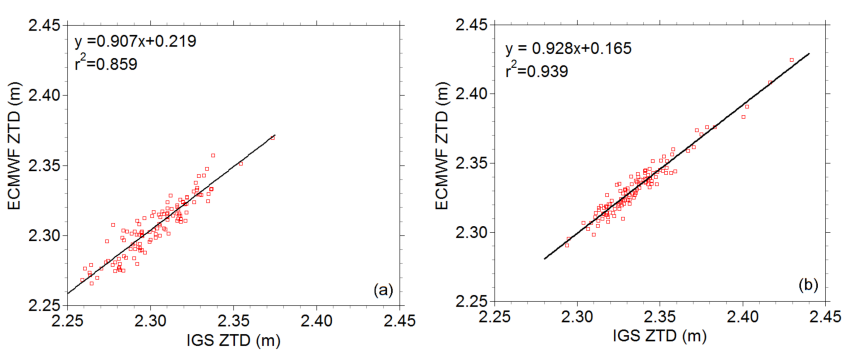

Figure 3. Scattergram of ECMWF and IGS ZTDs at stations KIRU (a) and NNOR (b). The vertical and horizontal axes show ECMWF and IGS ZTDs (m), respectively. The correlation coefficients $(r)$ and the results of a linear regression are also displayed.
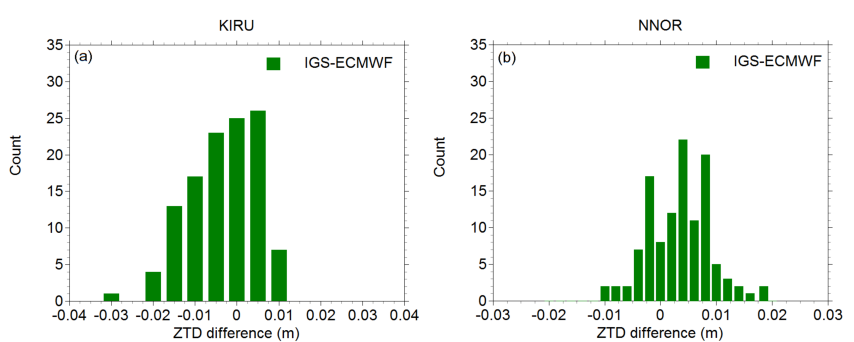

Figure 4. Distribution of ZTD differences between ECMWF and IGS at stations KIRU (a) and NNOR (b) for DOY 244-272, 2015.

a good agreement between the two solutions. Likewise, the rms values present a significant latitude dependence, which is smaller for high-latitude stations and larger for low-latitude stations, resulting from the distribution of atmospheric water vapor content with respect to the stations' latitudes. The rms values for stations in high-latitude regions are generally below $15 \mathrm{~mm}$, while the ones for the stations in low-latitude regions can reach up to $22 \mathrm{~mm}$. For an enhanced perspective, the rms values of ZWD differences between ECMWF and IGS are shown as a function of the geographical latitudes in Fig. 6, where a fitted parabola is also displayed in black. It can be clearly seen that the rms values reveal strong dependence on geographical latitudes, which are larger in lowlatitude (moist) regions and smaller in high-latitude (dry) regions. Similar findings were demonstrated in Dousa and Bennitt (2013), where a strong latitudinal dependency of ZTDs from the UK Met Office global model with respect to GPS ZTDs was described.

\subsection{Multi-GNSS PPP results}

To investigate the performance of applying tropospheric delay parameters derived from ECMWF into multi-GNSS PPP, two PPP scenarios including the standard PPP and the NWM-constrained PPP are carried out for comparison and validation, following the data processing algorithms presented in Sect. 2.3. Observational data from stations of the IGS MGEX network (see Fig. 1) in September 2015 are con- 

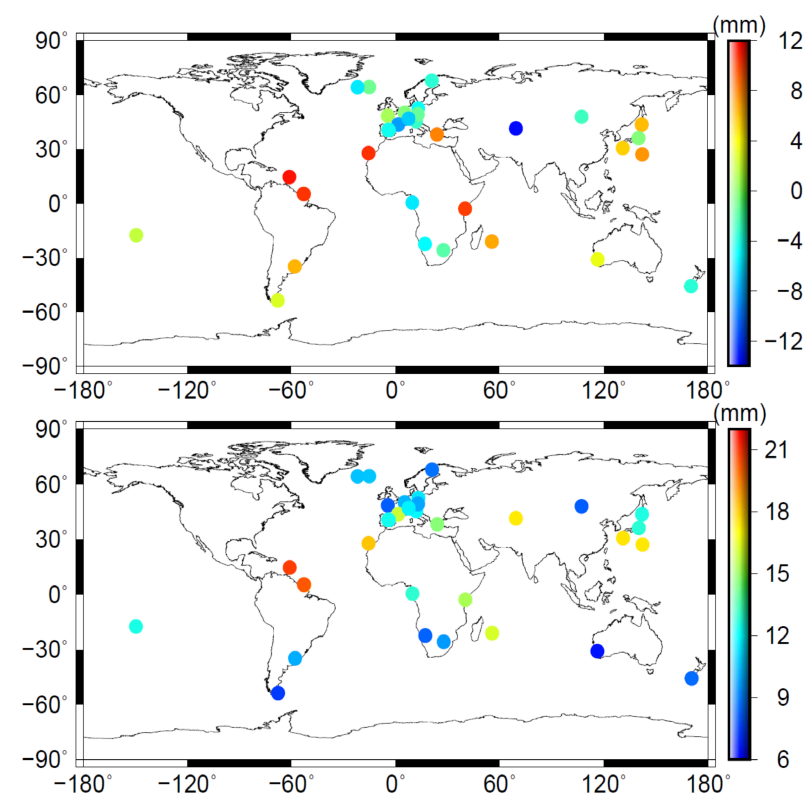

Figure 5. The map of the station-specific mean biases (top) and rms values (bottom) of ZTD differences between ECMWF and IGS for DOY 244-272, 2015.

sidered in this study. The post-processing weekly solution is used as the reference position. The convergence time is defined as the time required for the horizontal components to be better than $10 \mathrm{~cm}$, and the one needed for the vertical component to be better than $20 \mathrm{~cm}$.

As an example, Fig. 7 illustrates the estimated north, east, and vertical coordinates obtained from the two multiGNSS PPP processing methods at station WIND (Windhoek, Namibia; $22.57^{\circ} \mathrm{S}, 17.09^{\circ} \mathrm{E}$ ) on 12 September 2015. As a reference, positioning results derived from the stand-alone GPS PPP are also displayed applying similar strategies as the multi-GNSS processing. The standard PPP solutions are shown by black triangles, while the NWM-constrained PPP solutions are shown by red squares. The left figures show the multi-GNSS results. It can be seen that it takes about $17 \mathrm{~min}$ for the NWM-constrained multi-GNSS PPP to achieve an accuracy of better than $10 \mathrm{~cm}$ for the north component, in comparison to $25 \mathrm{~min}$ in the case of the standard PPP solution. The convergence time is shortened by about $32.0 \%$ by using the NWM-derived tropospheric delay parameters. Meanwhile, the positioning series of the standard PPP solution show a larger jump than that of the NWM-constrained PPP solution.

As for the east component, a convergence time of about $40 \mathrm{~min}$ for the standard vs. 25 min for the NWM-constrained PPP solution is noticed. Accordingly, the solution convergence is improved by about $37.5 \%$ with the NWMconstrained PPP. For the vertical component, a convergence time of about 20 and $15 \mathrm{~min}$ is required for the standard PPP solution and the NWM-constrained PPP solution, re-

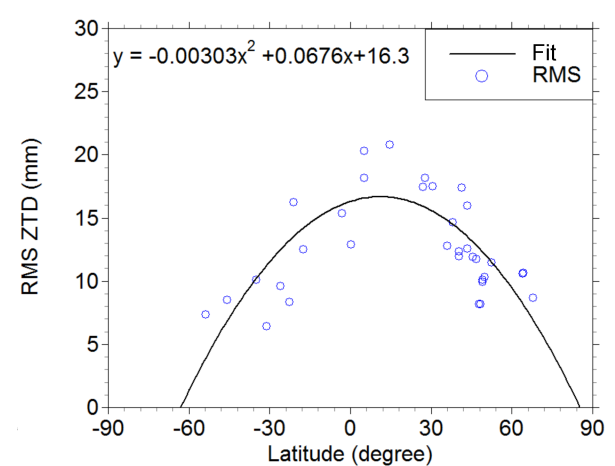

Figure 6. The rms values of ZTD differences between ECMWF and IGS as a function of geographical latitudes. A fitted second-order polynomial is also shown in black.

spectively, indicating an improvement of about $25.0 \%$ when applying the NWM-constrained PPP. Besides, the positioning series exhibit many more jumps and fluctuations with the standard PPP solution, in particular before the solution convergence, which was significantly improved when the NWMconstrained PPP is performed.

As shown in the right figures, for the standard GPS PPP, an accuracy of better than $10 \mathrm{~cm}$ is obtained after about 50 and $60 \mathrm{~min}$ for the north and east components, respectively. In comparison, it takes about 20 and $40 \mathrm{~min}$ for the NWM-constrained GPS PPP solution to become converged for the north and east components, shortening the solution convergence time by about 60.0 and $33.3 \%$. In the NWMconstrained GPS PPP solution, a convergence time of about $10 \mathrm{~min}$ is required for the vertical component, in comparison to $50 \mathrm{~min}$ in the case of the standard GPS PPP solution, revealing an improvement of up to $80.0 \%$. Moreover, it can be found that the NWM-constrained PPP reveals significant contribution to improving the positioning series of all three components, showing more stable and less fluctuated results. Furthermore, it is noteworthy that the positioning performance, not only the convergence time but also the positioning series of the GPS-only solution (right figures), becomes remarkably improved with the multi-GNSS processing (left figures). In addition, the corresponding summary over the solution improvements of the NWM-constrained PPP with respect to the standard PPP for both multi-GNSS and GPS solutions is listed in Table 1.

In Fig. 8, the statistical results of the multi-GNSS PPP solutions are presented with different session lengths $(5,8,10$, $15,17,20,25,30,40,50$, and $60 \mathrm{~min}$ ). The rms values of the positioning results for the north, east, and vertical components are calculated for all 21 four-system stations from the MGEX network over a sample period from 1 to 30 September 2015. The standard PPP solution is shown in orange, the NWM-constrained PPP solution in green. Obviously, the positioning accuracy of each component improves along with the increase of the session length for both PPP scenarios. In 

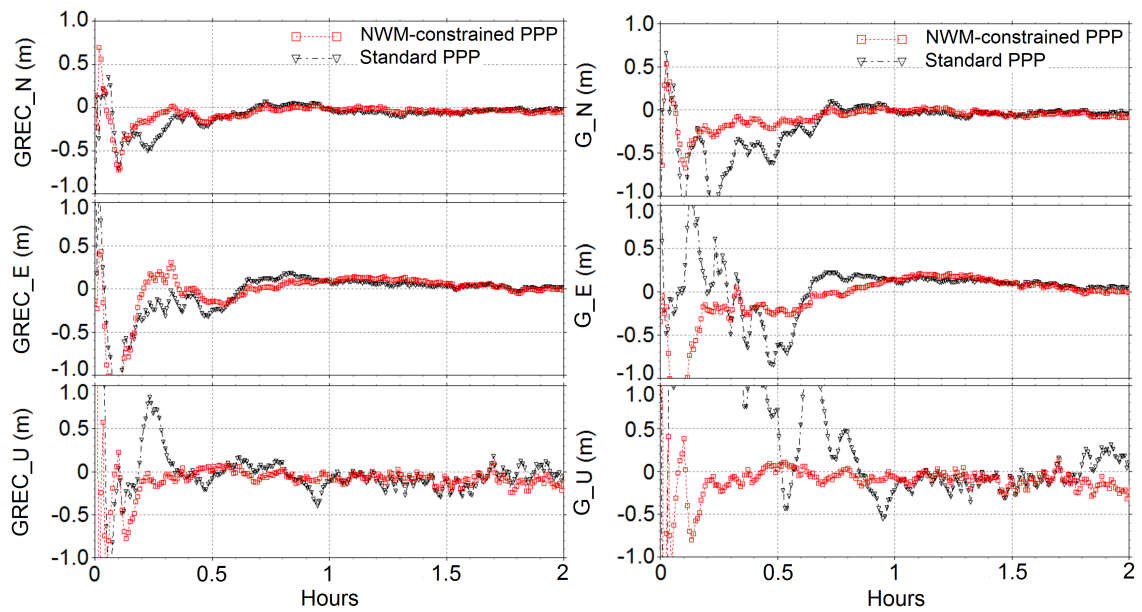

Figure 7. The multi-GNSS PPP (GREC) solution (left) and the stand-alone GPS PPP (G) solution (right) at station WIND (Windhoek, Namibia; $22.57^{\circ} \mathrm{S}, 17.09^{\circ} \mathrm{E}$ ) on 12 September 2015 (DOY 255 of 2015). The standard PPP solutions are shown by black triangles, while the NWM-constrained PPP solutions are shown by red squares.

Table 1. The summary over the solution improvements of the NWM-constrained PPP with respect to the standard PPP for both multi-GNSS and GPS solutions.

\begin{tabular}{lrrr|rrr|rrr}
\hline & \multicolumn{3}{c|}{ Standard PPP } & \multicolumn{3}{c}{ NWM-constrained PPP } & \multicolumn{4}{|c}{ Improvement } \\
& $N$ & $E$ & $U$ & $N$ & $E$ & $U$ & $N$ & $E$ & $U$ \\
& $(\mathrm{~min})$ & $(\mathrm{min})$ & $(\mathrm{min})$ & $(\mathrm{min})$ & $(\mathrm{min})$ & $(\mathrm{min})$ & & & \\
\hline Multi-GNSS & 25 & 40 & 20 & 17 & 25 & 15 & $32.0 \%$ & $37.5 \%$ & $25.0 \%$ \\
GPS & 50 & 60 & 50 & 20 & 40 & 10 & $60.0 \%$ & $33.3 \%$ & $80.0 \%$ \\
\hline
\end{tabular}

general, the positioning accuracy of the north component is better than that of the east and the vertical components, while the vertical component performs the worst, which may be attributed to the configuration of the satellite constellation.

For the north component, the rms values obtained from the NWM-constrained PPP solution are smaller than the ones from the standard PPP solution at the same session length, especially before the convergence. The positioning accuracy for the north component achieved with the NWMconstrained PPP is improved by about $2.5 \%$ compared to the one with the standard PPP. Besides, a convergence time of about 20 and $25 \mathrm{~min}$ is observed for the NWM-constrained PPP solution and the standard PPP solution, respectively: an improvement of about $20.0 \%$. In terms of the east component, higher accuracy can be found again for the NWMconstrained PPP solution, with the rms values reduced by about $12.1 \%$. Meanwhile, the NWM-constrained PPP solution takes about $17 \mathrm{~min}$ to become converged in comparison to $25 \mathrm{~min}$ for the standard PPP solution, a significant reduction of about $32.0 \%$ in the convergence time.

As for the vertical component, it can be noticed that the positioning accuracy achieved from the NWM-constrained PPP solution is obviously higher than that from the standard PPP solution, an improvement of about $18.7 \%$. More than $20 \mathrm{~min}$ are required for the standard PPP solution to obtain conver- gence, while the NWM-constrained PPP solution becomes converged in less than $15 \mathrm{~min}$, indicating an improvement of more than $25.0 \%$.

\section{Conclusions}

We developed a NWM-constrained PPP processing system where tropospheric delay parameters derived from the ECMWF analysis were applied to multi-GNSS precise positioning. Observations of stations from the IGS MGEX network were processed, with both standard PPP and the developed NWM-constrained PPP algorithm. The accuracy of the tropospheric delays derived from ECMWF was assessed through comparisons with the IGS final tropospheric delay products at all IGS MGEX stations. The positioning performance, including convergence time and positioning accuracy, achieved with the NWM-constrained PPP were investigated. The benefits of applying tropospheric delay parameters from the NWM to improve multi-GNSS PPP were demonstrated by comparing with the standard PPP solution.

Our results show that the mean biases between the ECMWF and IGS ZTDs are within $\pm 15 \mathrm{~mm}$, while the rms values of the ZTD differences are less than $22 \mathrm{~mm}$, indicating a good agreement between the two solutions. Besides, a better agreement for the high-latitude stations than for the 

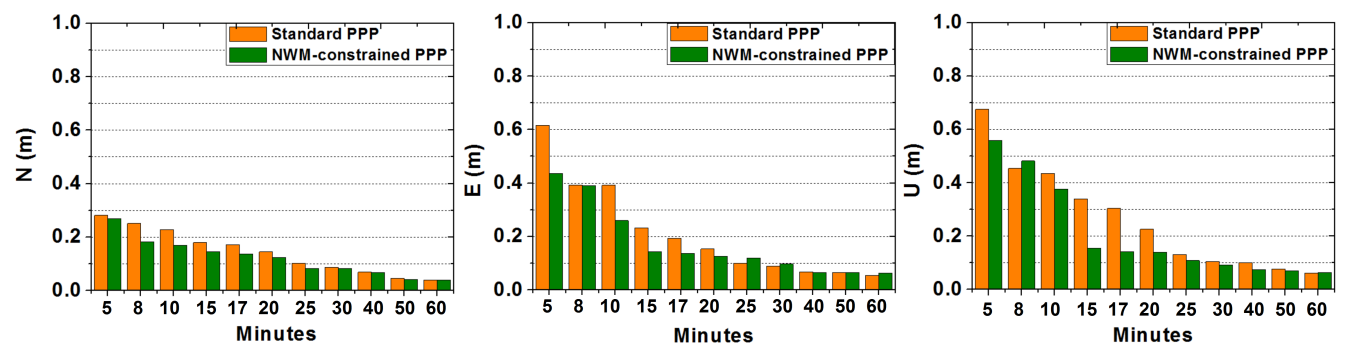

Figure 8. The rms values for the north, east, and vertical components with multi-GNSS PPP solution, shown at different session lengths $(5,8,10,15,17,20,25,30,40,50$, and $60 \mathrm{~min})$ for all 21 four-system stations of the MGEX network from 1 to 30 September 2015 . The standard PPP solution is shown in orange, the NWM-constrained PPP solution in green.

low-latitude stations can be noticed. Both the mean biases and rms values are smaller for high-latitude (dry) regions and larger for low-latitude (moist) regions, revealing significant latitude dependence. These may be accounted for by the distribution of atmospheric water vapor with respect to station latitudes. Furthermore, most of the peaks in the ZTD series, which are attributed to the rapid changes of the water vapor content above a given station, can be captured by both ECMWF and IGS solutions.

For the north component, it takes about $20 \mathrm{~min}$ for the NWM-constrained multi-GNSS PPP to achieve convergence, in comparison to $25 \mathrm{~min}$ for the standard PPP solution, showing a reduction of about $20.0 \%$ in the convergence time. An accuracy of better than $10 \mathrm{~cm}$ is achieved for the east component after a convergence time of about 25 and $17 \mathrm{~min}$ from the standard and the NWM-constrained PPP solutions, respectively. The convergence time is shortened by $32.0 \%$ with the NWM-constrained PPP. For the vertical component, a convergence time of about 20 and $15 \mathrm{~min}$ is required for the standard PPP solution and the NWM-constrained PPP solution, respectively, indicating an improvement of about $25.0 \%$ when applying the NWM-constrained PPP. Meanwhile, the positioning accuracy obtained from the NWM-constrained multi-GNSS PPP solution is also improved in comparison with the standard PPP solution after the same session length, in particular before the convergence. An improvement of positioning accuracy resulting from the NWM-constrained PPP solution of about $2.5,12.1$, and $18.7 \%$ for the north, east, and vertical components, respectively, can be found.

Besides, the positioning performance of the NWMconstrained GPS PPP solution achieves remarkable improvement compared to that of the standard GPS PPP solution, with the convergence time shortened by $60.0,33.3$, and $80.0 \%$ for the north, east, and vertical components, respectively, as well as more stable and less fluctuated positioning results for each coordinate component. Based on these results, it can be concluded that the performance of precise positioning benefits greatly from the multi-GNSS fusion in comparison to the stand-alone GPS solution, which can be further improved when the tropospheric delay parameters de- rived from NWM are implemented to the multi-GNSS PPP processing.

In future studies, we will investigate the performance of applying tropospheric delay parameters derived from the NWM into precise positioning with other single satellite navigation systems, such as the Russian GLObal NAvigation Satellite System (GLONASS) and the Chinese BeiDou Navigation Satellite System (BDS). Another research focus is the evaluation of the accuracy and performance of different numerical weather models, in order to find the most appropriate one to improve precise GNSS positioning.

\section{Data availability}

The GNSS observations were obtained from the IGS MGEX project, available at http://igs.org/mgex (Montenbruck et al., 2014). The IGS tropospheric products can be accessed at ftp://cddis.gsfc.nasa.gov/gnss/products/ troposphere/zpd. The ECMWF data (http://www/ecmwf.int/) are provided to GFZ by the German Weather Service.

Acknowledgements. Many thanks go to the International GNSS Service (IGS) for providing multi-GNSS data and the IGS final tropospheric products. The ECMWF data are provided to GFZ via the German Weather Service (DWD). Cuixian Lu is supported by the China Scholarship Council, which is gratefully acknowledged.

The article processing charges for this open-access

publication were covered by a Research

Centre of the Helmholtz Association.

Edited by: O. Bock

Reviewed by: two anonymous referees

\section{References}

Andrei, C. and Chen, R.: Assessment of time-series of troposphere zenith delays derived from the global data assimilation system numerical weather model, GPS Solut., 13, 109-117, 2008. 
Blewitt, G., Kreemer, C., Hammond, W. C., Plag, H.-P., Stein, S., and Okal, E.: Rapid determination of earthquake magnitude using GPS for tsunami warning systems, Geophys. Res. Lett., 33, L11309, doi:10.1029/2006GL026145, 2006.

Boehm, J., Niell, A., Tregoning, P., and Schuh, H.: Global Mapping Function (GMF): A new empirical mapping function based on numerical weather model data, Geophys. Res. Lett., 33, L7304, doi:10.1029/2005GL025546, 2006.

Byram, S., Hackman, C., and Tracey, J.: Computation of a HighPrecision GPS-Based Troposphere Product by the USNO, Proc. ION GNSS 2011, 19-23 September 2011, Portland, Oregon, USA, 572-578, 2011.

Chen, G. and Herring, T. A.: Effects of atmospheric azimuth asymmetry on the analysis of space geodetic data, J. Geophys. Res., 102, 20489-20502, doi:10.1029/97JB01739, 1997.

Dousa, J. and Bennitt, G. V.: Estimation and evaluation of hourly updated global GPS zenith total delays over ten months, GPS Solut., 17, 453-464, 2013.

Gao, Y. and Shen, X.: Improving ambiguity convergence in carrier phase-based precise point positioning, in: Proceedings of ION GPS-2001, 11-14 September, Salt Lake City, USA, 1532-1539, 2001.

Ge, M., Gendt, G., Rothacher, M., Shi, C., and Liu, J.: Resolution of GPS carrier-phase ambiguities in precise point positioning (PPP) with daily observations, J. Geodesy, 82, 389-399, doi:10.1007/s00190-007-0187-4, 2008.

Ge, M., Zhang, H., Jia, X., Song, S., and Wickert, J.: What Is Achievable with the Current COMPASS Constellation?, GPS World, 11, 29-34, 2012.

Hobiger, T., Ichikawa, R., Takasu, T., Koyama, Y., and Kondo, T.: Ray-traced troposphere slant delays for precise point positioning, Earth Planets Space, 60, e1-e4, 2008a.

Hobiger, T., Ichikawa, R., Koyama, Y., and Kondo, T.: Fast and accurate ray-tracing algorithms for real-time space geodetic applications using numerical weather models, J. Geophys. Res., 113, D20302, doi:10.1029/2008JD010503, 2008b.

Ibrahim, H. and El-Rabbany, A.: Performance analysis of NOAA tropospheric signal delay model, Meas. Sci. Technol., 22, 115107, doi:10.1088/0957-0233/22/11/115107, 2011.

Kouba, J.: A Guide to Using International GNSS Service (IGS) Products, available at: http://igscb.jpl.nasa.gov/igscb/resource/ pubs/UsingIGSProductsVer21.pdf (last access: 18 March 2016), 2009

Kouba, J. and Héroux, P.: Precise point positioning using IGS orbit and clock products, GPS Solut., 5, 12-28, doi:10.1007/PL00012883, 2001.

Lagler, K., Schindelegger, M., Boehm, J., Krásná, H., and Nilsson, T.: GPT2: Empirical slant delay model for radio space geodetic techniques, Geophys. Res. Lett., 40, 1069-1073, doi:10.1002/grl.50288, 2013.

Larson, K., Bodin, P., and Gomberg, J.: Using $1 \mathrm{~Hz}$ GPS data to measure deformations caused by the Denali fault earthquake, Science, 300, 1421-1424, 2003.

Li, X., Ge, M., Zhang, Y., Wang, R., Xu, P., Wickert, J., and Schuh, H.: New approach for earthquake/tsunami monitoring using dense GPS networks, Sci. Rep., 3, 2682, doi:10.1038/srep02682, 2013.
Li, X, Zhang, X., Ren, X., Fritsche, M., Wickert, J., and Schuh, H.: Precise positioning with current multi-constellation Global Navigation Satellite Systems: GPS, GLONASS, Galileo and BeiDou, Sci. Rep., 5, 8328, doi:10.1038/srep08328, 2015 a.

Li, X. Ge, M., Dai, X., Ren, X., Fritsche, M., Wickert, J., and Schuh, H.: Accuracy and reliability of multi-GNSS real-time precise positioning: GPS, GLONASS, BeiDou, and Galileo, J. Geodesy, 89, 607-635, doi:10.1007/s00190-015-0802-8, 2015b.

Li, X., Zus, F., Lu, C., Dick, G., Ning, T., Ge, M., Wickert, J., and Schuh, H.: Retrieving of atmospheric parameters from multiGNSS in real time: Validation with water vapor radiometer and numerical weather model, J. Geophys. Res.-Atmos., 120, 7189 7204, doi:10.1002/2015JD023454, 2015c.

Lu, C., Li, X., Nilsson, T., Ning, T., Heinkelmann, R., Ge, M., Glaser, S., and Schuh, H.: Real-time retrieval of precipitable water vapor from GPS and BeiDou observations, J. Geodesy, 89, 843-856, doi:10.1007/s00190-015-0818-0, 2015.

Lu, C., Li, X., Li, Z., Heinkelmann, R., Nilsson, T., Dick, G., Ge, M., and Schuh, H.: GNSS tropospheric gradients with high temporal resolution and their effect on precise positioning, J. Geophys. Res.-Atmos., 121, 912-930, doi:10.1002/2015JD024255, 2016.

Montenbruck, O., Steigenberger, P., Khachikyan, R., Weber, G., Langley, R. B., Mervart, L., and Hugentobler, U.: IGS-MGEX: preparing the ground for multi-constellation GNSS science, Inside GNSS, 9, 42-49, 2014.

Niell, A. E.: Global mapping functions for the atmosphere delay at radio wavelengths, J. Geophys. Res., 101, 3227-3246, 1996.

Pany, T., Pesec, P., and Stangl, G.: Elimination of tropospheric path delays in GPS observations with the ECMWF numerical weather model, Phys. Chem. Earth Pt. A, 26, 487-492, 2001.

Saastamoinen, J.: Contributions to the theory of atmospheric refraction - Part II. Refraction corrections in satellite geodesy, B. Géod., 47, 13-34, doi:10.1007/BF02522083, 1973.

Zhang, X. and Andersen, O.: Surface ice flow velocity and tide retrieval of the amery ice shelf using precise point positioning, J. Geodesy, 80, 171-176, doi:10.1007/s00190-006-0062-8, 2006.

Zumberge, J. F., Heflin, M. B., Jefferson, D. C., Watkins, M. M., and Webb, F. H.: Precise point positioning for the efficient and robust analysis of GPS data from large networks, J. Geophys. Res., 102, 5005-5017, 1997.

Zus, F., Dick, G., Dousa, J., Heise, S., and Wickert, J.: The rapid and precise computation of GPS slant total delays and mapping factors utilizing a numerical weather model, Radio Sci., 49, 207216, doi:10.1002/2013RS005280, 2014.

Zus, F., Dick, G., Dousa, J., and Wickert, J.: Systematic errors of mapping functions which are based on the VMF1 concept, GPS Solut., 19, 277-286, 2015a.

Zus, F., Dick, G., Heise, S., and Wickert, J.: A forward operator and its adjoint for GPS slant total delays, Radio Sci., 50, 393-405, doi:10.1002/2014RS005584, 2015b. 